\title{
ANALYTIC TOEPLITZ OPERATORS WITH AUTOMORPHIC SYMBOL.II
}

\author{
M. B. ABRAHAMSE AND JOSEPH A. BALL ${ }^{1}$
}

\begin{abstract}
For $\phi$ in $H^{\infty}$, let $T_{\phi}$ be the analytic Toeplitz operator with symbol $\phi$ and let $\left\{T_{\phi}\right\}^{\prime}$ be the commutant of $T_{\phi}$. Two infinite Blaschke products $\phi$ and $\psi$ are exhibited such that $\left\{T_{\phi}\right\}^{\prime} \cap\left\{T_{\psi}\right\}^{\prime}$ is not equal to $\left\{T_{\theta}\right\}^{\prime}$ for any inner function $\theta$. Also, two questions on reducing subspaces of analytic Toeplitz operators are answered.
\end{abstract}

1. Introduction. For $\phi$ in $H^{\infty}$ of the unit disk, the analytic Toeplitz operator $T_{\phi}$ on $H^{2}$ is defined by $T_{\phi}(f)=\phi f$. The commutant of $T_{\phi}$ is the set of operators $S$ on $H^{2}$ such that $S T_{\phi}=T_{\phi} S$ and is denoted $\left\{T_{\phi}\right\}^{\prime}$. In [4], Deddens and Wong ask the following question.

Question 1. Suppose $\left\{\chi_{\alpha}: \alpha\right.$ in $\left.\mathscr{Q}\right\}$ is a family of inner functions. Is $\cap_{\alpha \in \mathbb{Q}}\left\{T_{\chi_{\alpha}}\right\}^{\prime}$ equal to $\left\{T_{\theta}\right\}^{\prime}$ where $\theta$ is some inner function of which each $\chi_{\alpha}$ is a function?

James Thomson has shown that if one of the $\chi_{\alpha}$ is a finite Blaschke product, then the answer to Question 1 is affirmative [13]. In this paper it is shown that Thomson's result is sharp. In fact, we produce two infinite Blaschke products $\phi$ and $\psi$ such that $\left\{T_{\phi}\right\}^{\prime} \cap\left\{T_{\psi}\right\}^{\prime}$ does not equal $\left\{T_{\theta}\right\}^{\prime}$ for any inner function $\theta$.

The second author has raised the following two questions on reducing subspaces of analytic Toeplitz operators [3].

Question 2. If $\left\{\chi_{\alpha}: \alpha\right.$ in $\left.\mathcal{Q}\right\}$ is a collection of inner functions, if $\chi$ is the greatest common divisor of the $\chi_{\alpha}$, and if $\mathscr{N}$ is a closed subspace of $H^{2}$ which reduces each $T_{\chi_{a}}$, must $\mathfrak{N}$ reduce $T_{\chi}$ ?

Question 3. If $\phi$ in $H^{\infty}$ has inner-outer factorization $\phi=\chi F$ and if $\mathscr{M}$ is a closed subspace of $H^{2}$ which reduces $T_{\phi}$, must $\mathfrak{O}$ reduce $T_{\chi}$ and $T_{F}$ ?

It is shown that the status of Question 2 is the same as that of Question 1: the answer is affirmative if one of the $\chi_{\alpha}$ is a finite Blaschke product and there is a pair of infinite Blaschke products for which the answer is negative. The answer to Question 3 is also shown to be negative.

The counterexample for Question 1 makes use of the theory of bundle shifts developed by the first author and R. G. Douglas [2] and applied

Received by the editors January 26, 1976 and, in revised form, April 1, 1976.

AMS (MOS) subject classifications (1970). Primary 47B35, 47B20, $30 \mathrm{~A} 58$.

Key words and phrases. Toeplitz operator, automorphic function, modulus automorphic function, universal covering map, composition operator.

${ }^{1}$ The first author was supported in part by NSF Grant MPS-75-04594. 
previously to analytic Toeplitz operators by the first author [1]. It also makes use of a result of Rudin [9] and Stout [12] on inner generators of the space of rational functions on an annulus. The proof of the affirmative part of the answer to Question 2 uses the aforementioned result of Thomson [13] and the counterexamples for Questions 2 and 3 make use of a composition operator as in [1]. Here, the composition operator is preceded by a multiplication operator that makes the product unitary and the reducing subspace is the range of a projection in the $W^{*}$-algebra generated by this unitary. This technique of perturbing a composition operator to make it unitary was suggested to the first author several years ago by R. G. Douglas.

2. Automorphic inner functions. Let $D$ denote the unit disk $\{z:|z|<1\}$, let $R$ denote the annulus $\left\{z: \frac{1}{2}<|z|<1\right\}$, and let $\pi$ be the holomorphic universal covering map from $D$ onto $R$ as defined in [11] and [1] by

$$
\pi(z)=\exp \left(\frac{i}{\pi} \log \frac{1}{2} \log \frac{1+z}{1-z}+\frac{1}{2} \log \frac{1}{2}\right)
$$

where Log is the principal branch of the logarithm. It is shown in [1] that $T_{\pi}$ is a pure subnormal operator with spectrum contained in the closure of $R$ and normal spectrum contained in the boundary of $R$ and thus, by [2, Theorem 11], there is a vector bundle $E$ over $R$ such that the bundle shift $S_{E}$ is unitarily equivalent to $T_{\pi}$. The bundle shift $S_{E}$ is multiplication by $z$ on the space $H_{E}^{2}(R)$ of $H^{2}$ cross-sections of the bundle $E$. Let $A(R)$ be the space of continuous functions on the closure of $R$ that can be approximated uniformly by rational functions with poles off the closure of $R$ and for $\phi$ in $A(R)$ let $T_{\phi}^{E}$ be the operator on $H_{E}^{2}(R)$ defined by $T_{\phi}^{E}(f)=\phi f$. It is easily verified that $\phi\left(T_{\pi}\right)=T_{\phi \circ \pi}$ and $\phi\left(S_{E}\right)=T_{\phi}^{E}$ for all $\phi$ in $A(R)$. This establishes the following lemma.

LEMMA 2.1. There is a unitary operator $V$ from $H^{2}(D)$ onto $H_{E}^{2}(R)$ such that $V T_{\phi \circ \pi}=T_{\phi}^{E} V$ for all $\phi$ in $A(R)$.

A generating set for $A(R)$ is a subset $G$ of $A(R)$ such that the smallest uniformly closed subalgebra of $A(R)$ containing $G$ is all of $A(R)$. The space $H^{\infty}(R)$ is the Banach algebra of all bounded analytic functions on $R$. For a set of operators $S$, the second commutant of $S$ is the commutant of the commutant of $\varsigma$ and is denoted $\varsigma "$.

LEMma 2.2. If $G$ is a generating set for $A(R)$, then the Banach algebra $\left\{T_{\phi \circ \pi}: \phi \text { in } G\right\}^{\prime \prime}$ is isomorphic to $H^{\infty}(R)$.

Proof. By Lemma 2.1, the algebra $\left\{T_{\phi \circ \pi}: \phi \text { in } G\right\}^{\prime \prime}$ is unitarily equivalent to the algebra $\left\{T_{\phi}^{E}: \phi \text { in } G\right\}^{\prime \prime}$. Since $G$ is a generating set for $A(R)$, the latter algebra is equal to the second commutant of the bundle shift $S_{E}$. The result now follows from [2, Theorem 4].

The function in $A(R)$ is said to be inner if it is unimodular on the boundary of $R$. In the following lemma and elsewhere in this paper, a 
function which is a Blaschke product times a scalar of unit modulus shall be referred to as a Blaschke product.

LEMMA 2.3. If $\phi$ is a nonconstant inner function in $A(R)$, then $\phi \circ \pi$ is an infinite Blaschke product.

Proof. The covering map $\pi$ is continuous on the set $\{z:|z| \leqslant 1, z \neq 1$, $z \neq-1\}$ and maps the sets $\{z:|z|=1, \operatorname{Im} z>0\}$ and $\{z:|z|=1, \operatorname{Im}$ $z<0\}$ onto the outer and inner boundaries of $R$ respectively. It follows that $\phi \circ \pi$ is an inner function of the form

$$
\phi(\pi(z))=\lambda B(z) \exp \left(-a \frac{1+z}{1-z}\right) \exp \left(-b \frac{-1+z}{-1-z}\right)
$$

where $|\lambda|=1, B$ is a Blaschke product, and $a$ and $b$ are nonnegative real numbers. If $a$ is not equal to zero, then

$$
0=\lim _{x \uparrow 1} \phi(\pi(x))=\lim _{t \rightarrow \infty} \phi\left(e^{i t} / \sqrt{ } 2\right) .
$$

(Here one uses the fact that $\pi$ maps the interval $(-1,1)$ around the circle $\{|z|=1 / \sqrt{ } 2\}$ an infinite number of times.) It follows that $\phi\left(e^{i t} / \sqrt{ } 2\right)=0$ for all $t$, hence, the function $\phi$ is identically zero, a contradiction. Thus, $a=0$ and a similar argument shows that $b=0$. Thus, $\phi \circ \pi$ is a Blaschke product. Since $\phi$ is not constant, there is a point $\beta$ in $R$ such that $\phi(\beta)=0$ and therefore $\phi \circ \pi$ must vanish on the infinite set $\pi^{-1}(\beta)$. Thus, $\phi \circ \pi$ is an infinite Blaschke product.

THEOREM 1. There are two infinite Blaschke products $\phi$ and $\psi$ such that the Banach algebra $\left\{T_{\phi}, T_{\psi}\right\}^{\prime \prime}$ is isomorphic to $H^{\infty}(R)$.

Proof. It has been shown by Rudin that there are two inner functions $\phi_{1}$ and $\psi_{1}$ which form a separating pair for $A(R)$ [9]. It follows that the map $z \rightarrow\left(\phi_{1}(z), \psi_{1}(z)\right)$ is an embedding of the closure of $R$ into the closure of the polydisc $U^{2}=\left\{\left(z_{1}, z_{2}\right):\left|z_{1}\right|<1,\left|z_{2}\right|<1\right\}$ which takes the boundary of $R$ into the boundary of $U^{2}$. Let $V$ denote the range of this embedding. Stout has shown that every function $f$ in $A(R)$, when viewed as a function on $V$, can be extended to a continuous function $\tilde{f}$ on the closure of $U^{2}$ which is analytic on $U^{2}$ [12, Theorem II.1]. Let $\left\{p_{n}\right\}$ be a sequence of polynomials in two variables which converges uniformly to $\tilde{f}$ on the closure of $U^{2}$. Then the sequence $\left\{p_{n}\left(\phi_{1}, \psi_{1}\right)\right\}$ converges uniformly to $f$ on the closure of $R$ which proves that $\phi_{1}$ and $\psi_{1}$ generate $A(R)$. Set $\phi=\phi_{1} \circ \pi$ and $\psi=\psi_{1} \circ \pi$. The theorem now follows from Lemmas 2.2 and 2.3.

To see that Theorem 1 settles Question 1, suppose that $\theta$ is an inner function such that $\left\{T_{\phi}\right\}^{\prime} \cap\left\{T_{\psi}\right\}^{\prime}=\left\{T_{\theta}\right\}^{\prime}$. Then $\left\{T_{\phi}, T_{\psi}\right\}^{\prime \prime}=\left\{T_{\theta}\right\}^{\prime \prime}$. However, since $T_{\theta}$ is a unilateral shift, its double commutant $\left\{T_{\theta}\right\}^{\prime \prime}$ is isomorphic to the Banach algebra $H^{\infty}(D)$. Thus, according to Theorem 1, the Banach algebras $H^{\infty}(R)$ and $H^{\infty}(D)$ are isomorphic. This is a contradiction. In fact, if $R_{1}$ and $R_{2}$ are two bounded domains in the plane with analytic boundary and if $H^{\infty}\left(R_{1}\right)$ is isomorphic to $H^{\infty}\left(R_{2}\right)$, then $R_{1}$ and $R_{2}$ are conformally 
equivalent by a general result of Chevalley and Kakutani [10].

3. Reducing subspaces. We begin with an affirmative result.

THEOREM 2. If $\left\{\chi_{\alpha}: \alpha\right.$ in $\left.\hat{\mathcal{Q}}\right\}$ is a collection of inner functions which contains a finite Blaschke product, if $\chi$ is the greatest common divisor of the $\chi_{\alpha}$, and if $\mathrm{N} / \mathrm{C}$ is a closed subspace which reduces each $T_{\chi_{\alpha}}$, then 0 reduces $T_{\chi}$.

Proof. The hypotheses imply that there is a finite Blaschke product $\theta$ and inner functions $\psi_{\alpha}$ such that $\chi_{\alpha}=\psi_{\alpha} \circ \theta$ and $\left\{T_{\chi_{\alpha}}: \alpha \text { in } Q\right\}^{\prime}=\left\{T_{\theta}\right\}^{\prime}$ [13]. Set $\Psi=$ g.c.d. $\left\{\Psi_{\alpha}\right\}$. It follows that $\chi=\Psi \circ \theta$. In other words

$\left[\right.$ g.c.d. $\left\{\Psi_{\alpha}: \alpha\right.$ in $\left.\left.Q^{\prime}\right\}\right] \circ \theta=$ g.c.d. $\left\{\Psi_{\alpha} \circ \theta: \alpha\right.$ in $\left.\dot{G}^{\prime}\right\}$.

For the case here where the collection $\left\{\Psi_{\alpha}\right\}$ contains a finite Blaschke product, the function $\Psi$ is the Blaschke product vanishing precisely at the common zeroes (counting multiplicities) of the $\Psi_{\alpha}$. Since $\theta$ is also finite Blaschke, $\Psi \circ \theta$ is the finite Blaschke product which vanishes precisely at the common zeroes (counting multiplicities) of $\Psi_{\alpha} \circ \theta$, and hence $\Psi \circ \theta=\chi$. The general case of $(*)$ can be shown using Theorem 1 (iv) of [3]. Now suppose that $P$ is a projection which commutes with each $T_{\chi_{\alpha}}$. Then $P$ commutes with $T_{\theta}$ and hence with $\Psi\left(T_{\theta}\right)$. But $\Psi\left(T_{\theta}\right)=T_{\Psi \circ \theta}=T_{\chi}$ which proves the theorem.

Let $A$ be the linear fractional transformation which generates the covering group for $\pi$ as in [1]. Thus, $A$ maps the disk onto itself and a function $\phi$ on the disk is of the form $\Psi \circ \pi$ if and only if $\phi$ is automorphic with respect to $A$, that is, $\phi(A(z))=\phi(z)$ for all $z$ in $D$. The following lemma deals with a composition operator defined with respect to $A$ which is perturbed in such a way to make the result unitary. It also deals with functions modulus automorphic with respect to $A$. We now define these objects.

The composition operator $C_{A}$ on $H^{2}$ is defined by the equation $C_{A}(f)=$ $f \circ A$. It has been shown by Nordgren [7] that for $f$ in $H^{2}$,

$$
\left\|C_{A}(f)\right\|^{2}=\frac{1}{2 \pi} \int_{0}^{2 \pi}\left|f\left(e^{i \theta}\right)\right|^{2} \operatorname{Re}\left(\frac{e^{i \theta}+A(0)}{e^{i \theta}-A(0)}\right) d \theta .
$$

Thus, if $k_{A}$ is the outer function such that

$$
\left|k_{A}\left(e^{i \theta}\right)\right|^{2}=\left[\operatorname{Re}\left(\frac{e^{i \theta}+A(0)}{e^{i \theta}-A(0)}\right)\right]^{-1},
$$

then $k_{A}$ is an invertible element of $H^{\infty}$ and $\left\|C_{A}\left(k_{A} f\right)\right\|^{2}=\|f\|^{2}$ for all $f$ in $H^{2}$. Thus, the operator $U_{A}$ on $H^{2}$ defined by $U_{A}(f)=C_{A}\left(k_{A} f\right)$ is unitary. Let $\phi$ be in $H^{\infty}$ of the disk, and let $\lambda$ be a scalar of modulus one. The function $\phi$ is said to be modulus automorphic with respect to $A$ of index $\lambda$ if $\phi(A(z))=$ $\lambda \phi(z)$ for all $z$ in $D$.

Lemma 3.1. If $\phi$ is modulus automorphic with respect to $A$ of index $\lambda$, then 
$U_{A} T_{\phi}=\lambda T_{\phi} U_{A}$. Hence, the operator $U_{A}$ commutes with $T_{\phi}$ if and only if $\phi$ is automorphic with respect to $A$.

Proof. Evaluate.

Actually, Lemma 3.1 holds for an arbitrary linear fractional transformation which maps the disk onto itself.

THEOREM 3. There are two infinite Blaschke products $\phi$ and $\psi$ and a subspace M of $H^{2}$ such that $\mathfrak{N}$ reduces $T_{\phi}$ and $T_{\psi}$ and $\mathfrak{N}$ does not reduce $T_{\chi}$ where $\chi$ is the greatest common divisor of $\phi$ and $\psi$.

Proof. For $a$ in $R$, let $\phi_{a}$ be the Blaschke product for the set $\pi^{-1}(a)$. It has been shown by Sarason that $\phi_{a}$ is modulus automorphic with respect to $A$ of index $e^{2 \pi t i}$ where $t=\log |a| / \log 2[11, \mathrm{p}$. 18]. Thus, if $a=1 / \sqrt{ } 2$, then the Blaschke products $\phi=\phi_{a} \phi_{a}$ and $\psi=\phi_{a} \phi_{i a}$ are automorphic with respect to $A$ and their greatest common divisor $\chi=\phi_{a}$ is modulus automorphic with respect to $A$ of index -1 . Thus, by Lemma 3.1, the unitary operator $U_{A}$ commutes with $T_{\phi}$ and $T_{\psi}$ and does not commute with $T_{\chi}$. Since the projections in a $W^{*}$-algebra always generate the algebra, there is a projection $P$ in the $W^{*}$-algebra generated by $U_{A}$ such that $P$ does not commute with $T_{\chi}$. But this projection does commute with $T_{\phi}$ and $T_{\psi}$ which proves the theorem.

The following theorem is closely related to Theorem 3 of [1].

THEOREM 4. If $\phi(z)=\pi(z)-\frac{3}{4}$ and if $\phi=\chi F$ is the inner-outer factorization of $\phi$, then there is a reducing subspace for $T_{\phi}$ which reduces neither $T_{\chi}$ nor $T_{F}$.

Proof. The function $\chi$ is modulus automorphic and not automorphic with respect to $A$ (see the proof of Theorem 3 in [1]). By Lemma 3.1, the unitary operator $U_{A}$ does not commute with $T_{\chi}$ and thus there is a projection $P$ in the $W^{*}$-algebra generated by $U_{A}$ such that $P$ does not commute with $T_{\chi}$. Since $\phi$ is automorphic with respect to $A$, the operator $U_{A}$ commutes with $T_{\phi}$ by Lemma 3.1, and thus $P$ commutes with $T_{\phi}$. From the equations (1) $T_{\phi}=$ $T_{\chi} T_{F}$, (2) $T_{\phi} P=P T_{\phi}$, (3) $T_{\chi} P \neq P T_{\chi}$, and the fact that (4) $T_{F}$ is invertible, it follows that $T_{F} P \neq P T_{F}$. This completes the proof of Theorem 4.

4. Comments. The examples in Theorems 3 and 4 involve projections in the $W^{*}$-algebra generated by $U_{A}$ (sometimes called spectral projections for $U_{A}$ ). In fact, the operator $U_{A}$ is a bilateral shift of infinite multiplicity [5] and therefore the $W^{*}$-algebra generated by $U_{A}$ is $L^{\infty}$ of the unit circle.

The spectral subspaces for $U_{A}$ are reducing subspaces for $T_{\pi}$, a fact which gives a proof of Theorem 2 in [1] that does not invoke the theory of bundle shifts. The proof of the following proposition involves an analysis of the bundle $E$ of $\S 2$ and is omitted. Following Rosenthal [8], an operator $A$ is said to be completely reducible if for each nonzero reducing subspace $\mathscr{N}$, the operator $A \mid$ ON has a nontrivial reducing subspace.

PROPOSITION 4.1. The operator $T_{\pi}$ is completely reducible. 
This proposition suggests the following reformulation of a question of Nordgren [6] which was shown to be false in general by the first author [1].

Question. If $\phi$ is in $H^{\infty}$ and if $T_{\phi}$ has a nontrivial reducing subspace $\mathbb{R}$ such that $T_{\phi}$ OO is irreducible, must there be a function $\psi$ in $H^{\infty}$ and an inner function $\theta$ which is not a linear fractional transformation such that $\phi=\psi \circ \theta$ ?

In an abstract of his dissertation, Carl Cowen has announced an affirmative answer to Question 1 if for some $w$ in the unit disk, the greatest common divisor of $\left\{\chi_{\alpha}-\chi_{\alpha}(w) \mid \alpha\right.$ in $\left.Q\right\}$ is finite Blaschke. It follows that Theorem 2 remains true under this assumption.

The authors wish to acknowledge the referee for simplifying the proof of Theorem 5 and for pointing out the recent results of Lubin on the operator $U_{A}$.

\section{REFERENCES}

1. M. B. Abrahamse, Analytic Toeplitz operators with automorphic symbol, Proc. Amer. Math. Soc. 52 (1975), 297-302.

2. M. B. Abrahamse and R. G. Douglas, A class of subnormal operators related to multiply-connected domains, Advances in Math. 19 (1976), 1-43.

3. J. A. Ball, Hardy space expectation operators and reducing subspaces, Proc. Amer. Math. Soc. 47 (1975), 351-357. MR 50 \# 10887.

4. J. A. Deddens and T. K. Wong, The commutant of analytic Toeplitz operators, Trans. Amer. Math. Soc. 184 (1973), 261-273. MR 48 \#2819.

5. A. Lubin, Isometries induced by composition operators and invariant subspaces, Illinois $\mathrm{J}$. Math. 19 (1975), 424-427.

6. E. A. Nordgren, Reducing subspaces of analytic Toeplitz operators, Duke Math. J. 34 (1967), 175-181. MR 35 \#155.

7. , Composition operators, Canad. J. Math. 20 (1968), 442-449. MR 36 \#6961.

8. P. Rosenthal, Completely reducible operators, Proc. Amer. Math. Soc. 19 (1968), 826-830. MR $37 \# 6789$.

9. W. Rudin, Pairs of inner functions on finite Riemann surfaces, Trans. Amer. Math. Soc. 140 (1969), 423-434. MR 39 \#2968.

10. Some theorems on bounded analytic functions, Trans. Amer. Math. Soc. 78 (1955), 333-342. MR 16, 685 .

11. D. Sarason, The $H^{p}$ spaces of an annulus, Mem. Amer. Math. Soc. No. 56 (1965). MR 32 \#6256.

12. E. L. Stout, On some algebras of analytic functions on finite open Riemann surfaces, Math. Z. 92 (1966), 366-379. Correction, ibid. 95 (1967), 403-404. MR 34 \#358; 2913.

13. J. E. Thomson, Intersection of commutants of analytic Toeplitz operators, Proc. Amer. Math. Soc. 52 (1975), 305-310.

Department of Mathematics, University of Virginia, Charlottesville, Virginia 22901

Department of Mathematics, Virginia Polytechnic Institute and State University, BLACKSBURG, VIRGINIA 24061 